\title{
A Three Dimensional Performance Analysis of a Developed Evacuated Tube Collector using a CFD Fluent Solar Load Model
}

\author{
Basil. H. Ali 1,a, S. I. Gilani ${ }^{1}$ and Hussain H. Al-Kayiem ${ }^{1}$ \\ ${ }^{1}$ Mechanical Engineering Department, Universiti Teknologi PETRONAS, Malaysia
}

\begin{abstract}
For the flow through type selected geometry of the designed evacuated tube collector ETC, a three dimensional simulation and analysis of the thermal performance was done, using the ray-tracing solar load model provided by the ANSYS-FLUENT software. In this model the solar radiation heat flux is solved using fair weather condition radiation equations, and then solar radiation load is considered as a heat source term in the energy equation. The thermal effects of the fluid flow and heat transfer in the collector, temperature fields as well as the solar radiation distribution, and other related factors under the different conditions have been discussed and analyzed.
\end{abstract}

\section{Introduction}

The ordinary flat plate collectors are not practical for elevated temperature ranges due to their high heat loss coefficient. When high temperature applications are desired, the heat losses are required to be minimal, which possible to be accomplished by two methods; evacuation method and concentration method, either singly or in combination. The evacuated tube collectors (ETCs) can be achieved using selective coatings of the absorber, because with no coated absorber, radiation losses would dominate at high temperatures, and eliminating convection alone would not be very effective[1].

The high cost usually are involved in the experimental works, that's why a great work was done by the scientist to develop several dynamic procedures, and numerical approaches to lessening this huge expense and works. In the last year Computational Fluid-Dynamics (CFD) approach has become a powerful tool, to investigate the performance of several machines and appliances heat transfer phenomena. A lot of work has been done using both commercial and individual scientists developed codes, investigating several aspects concerning the heat transfer mechanism in a solar collector.

The main three methods of heat transfer, conduction, convection and radiation are applied in solar collector models, where the radiation comes from the sun is the main target in these thermal devices. ANSYS-FLUENT commercial software is providing five radiation simulation models, which allow inclusion of radiation, with or without a participating medium, in the heat transfer simulations.

Researchers used these models in several studies. Numerical simulation of a solar flat plate collector using the discrete transfer radiation model (DTRM) - A CFD Approach was provided by K. Vasudeva Karanth [2]. Khalid and H. Joined in 2012 perform a study to investigate the heat transfer behavior of a finned solar air collector using fluent[3]. In 2013 a CFD analysis of triangular absorber

\footnotetext{
${ }^{\text {a }}$ Corresponding author : basil4eng@yahoo.com
}

This is an Open Access article distributed under the terms of the Creative Commons Attribution License 2.0, which permits unrestricted use, distribution, and reproduction in any medium, provided the original work is properly cited. 
tube of a solar flat plate collector is done by Basavanna. S[4]. Another work in2013 was done by Arun Venu is a Simulation Studies on Porous Medium Integrated Dual Purpose Solar Collector [5]. This showed the importance of this tool.

The objective of this work is to develop a CFD numerical model that provides useful information to analyze the solar evacuated tube collector behavior, and to obtain the behavior of the developed ETS collector without performing the set of complicated and expensive experimental tests.

\section{CFD FLUENT modeling methodology}

The major steps involved in the simulation are, outlining and specifying of the model geometry according to the main design consideration. When CFD analysis tool is based on the finite element model the meshing of the model is an important second step of the solution. The setting of the general simulation model is done when the energy equation and radiation solar load model take place. Identify the model orientation, latitude, altitude, and Time zone. Specify model used material types, properties, and boundary conditions. Finally, setting the solution and post process are executed.

\subsection{Developed model}

Heating of surfaces due to radiation can be achieved in CFD model using Discrete Transfer Radiation Model, or P-1 Radiation Model, or Rosseland Radiation Model, or Surface-to-Surface (S2S) Radiation Model, or Discrete Ordinates (DO) Radiation Model. Also, ANSYS-FLUENT provides a solar load model that allows inclusion of the effects of solar radiation in the simulation [6].

The developed ETC collector is a flow through type, in which the flow domain consists of a copper absorber tube of $1.5 \mathrm{~m}$ length, with $25.4 \mathrm{~mm}$ inner diameter and $3 \mathrm{~mm}$ thickness. This circular absorber tube is insulated with circular glass enclosure with $76.2 \mathrm{~mm}$ inner diameter and $5 \mathrm{~mm}$ thickness. A $1 \mathrm{~mm}$ thickness selective absorber is covering the copper tube in the vacuum area between the glass and cooper tubes to improve tube absorptivity. The water enters the copper tube with $0.01 \mathrm{~m} / \mathrm{s}$ through the inlet face. Pressure outlet end is selected. Two copper to glass sealed covers are selected to enclose the vacuum area. The collector configuration is as shown in figure 1.

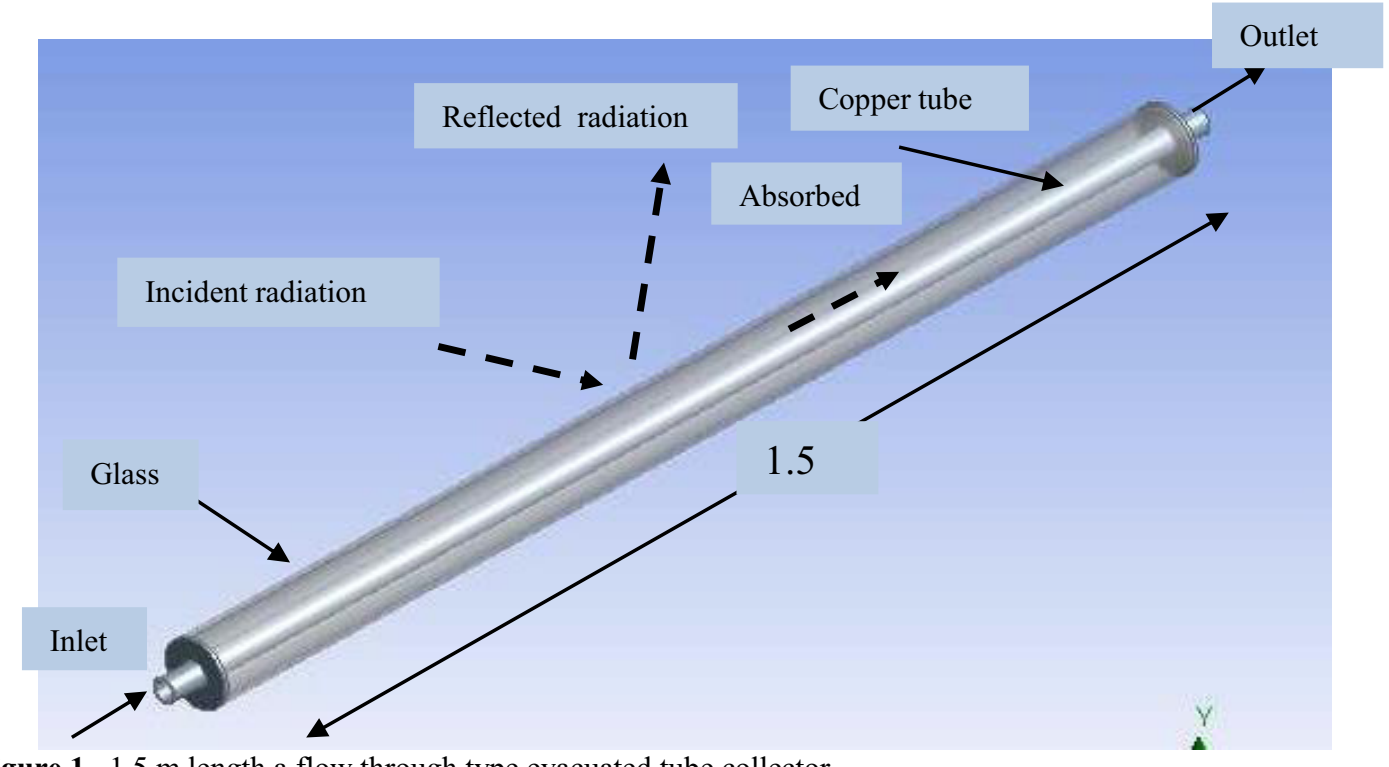

Figure 1. $1.5 \mathrm{~m}$ length a flow through type evacuated tube collector

The simulation is carried out under the following assumptions.

(1) The water flow is continuous and incompressible. 
(2) The flow is steady and laminar, as the velocity of flow is low.

(3) The thermo physical properties of the absorber tube.

(4) The bottom side of the absorber tube, covers, and the glass tube is assumed to be adiabatic.

(5) The selected longitude, latitude, and time zone are 100, 4, and +8 for the calculation of the sun direction vector, for the solar site located at Universiti Teknologi PETRONAS

(6) Solar ray tracing, fair weather condition model option is applied.

(7) Solar calculator is operated to calculate the required quantities of direct solar irradiation, diffuse solar irradiation, direct and IR absorptivity (opaque wall), direct and IR absorptivity and transmissivity (semi transparent wall and porous jump), diffuse hemispherical absorptivity and transmissivity (semi transparent wall)

(8) The value of spectral fraction and ground reflectivity are 0.5 and 0.3 respectively

(9) Solar transmissivity factors for the model materials are defined as follow

\subsection{Fair weather condition method}

Two computing options are provided by ANSYS FLUENT for solar load model, fair weather conditions method and theoretical maximum method. For coated glazing, the spectral transmissivity and reflectivity at any incident angle are approximated from the normal angle of incidence. Transmissivity, under that sense, is given by [6].

$$
\begin{gathered}
T(\theta, \lambda)=T(0, \lambda) \operatorname{Tref}(\theta) \\
\operatorname{Tref}(\theta)=a 0+a 1 \cos (\theta)+a^{2} \cos \left(\theta^{2}\right)+a^{3} \cos \left(\theta^{3}\right)+a^{4} \cos \left(\theta^{4}\right)
\end{gathered}
$$

And reflectivity is given by

$$
\begin{gathered}
R(\theta, \lambda)=R(0, \lambda)[1-\operatorname{Rref}(\theta)]+\operatorname{Rref}(\theta) \\
\operatorname{Rref}(\theta)=b 0+b 1 \cos (\theta)+b^{2} \cos \left(\theta^{2}\right)+b^{3} \cos \left(\theta^{3}\right)+b^{4} \cos \left(\theta^{4}\right)
\end{gathered}
$$

$T(0, \lambda)$ and $R(0, \lambda)$ are the normal transmissivity and reflectivity respectively, there values are specified in the wall boundary conditions dialog box.

Application of the equation for direct normal irradiation, for fair weather condition, is obtained from ASHREE handbook as.

$$
E d n=\frac{A}{B / e^{\sin \beta}}
$$

$A$ is the apparent solar irradiation at air mass, $m=0$.

$B$ is the atmospheric extinction coefficient.

$\beta$ is the solar altitude (in degrees) above the horizontal.

Diffused solar irradiation on a vertical surface is given by [2].

$$
E d=C \times Y \times E d n
$$

$C$ is a constant (the value is obtained from ASHREE handbook).

$Y$ is the ratio of sky diffuse radiation on vertical surface to that on horizontal surface.

$E d n$ is the direct normal irradiation at earth's surface on clear day.

For surfaces other than vertical, the diffuse solar irradiation is given by

$$
E d=C E d n \frac{(1+\cos \varepsilon)}{2}
$$


Where, $\varepsilon$ is the tilt angle of the surface in degree from horizontal plane.

And the equation for ground reflected solar irradiation on the surface is given by

$$
E r=E d n(C+\sin \beta) \rho_{g} \frac{(1-\cos \varepsilon)}{2}
$$

$\rho_{g}$ is the ground reflectivity. The total diffuse irradiation on a surface will be the sum of these values. The input for diffuse solar radiation is taken from the solar calculator.

\section{Simulation results}

For the CFD Fluent steady state simulation, the program is converged with the following input data. The initial velocity of water at the inlet of the copper tube is $0.01 \mathrm{~m} / \mathrm{s}$, and the initial pressure at the outlet is atmospheric. The boundary conditions for the glass, copper, water are selected for semi transparent wall with 0.1 degree of absorptivity and 0.8 degree of transmissivity. The interior water is defined as a porous jump medium, and 300 Kelvin initiates the simulation temperature, for 0.5 visible for the IR fraction of solar radiation at selected model location. Figure 2 and 3 indicate the convergence and contour distribution of model static temperature, and water velocity distribution respectively.
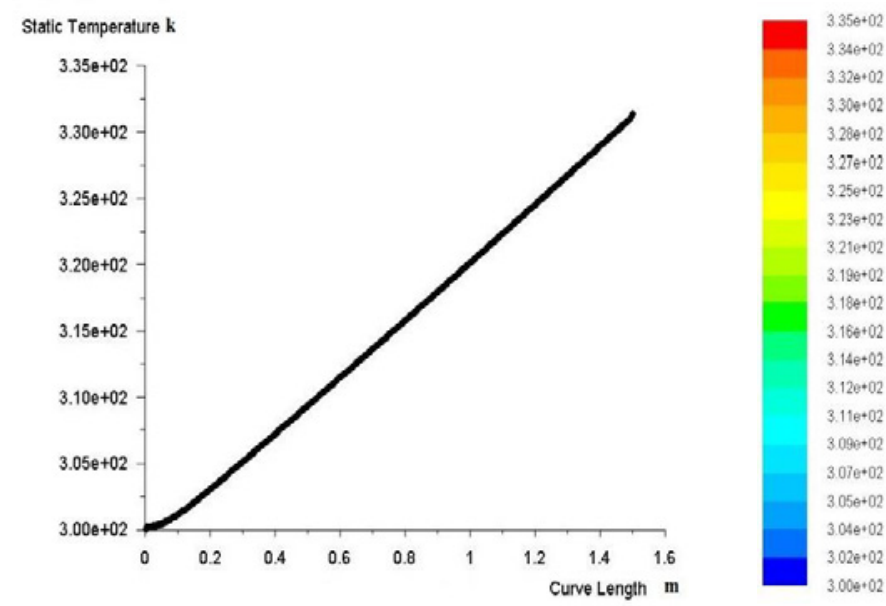

Figure 2. left outlet static temperature per length- right results contour of the temperature distribution
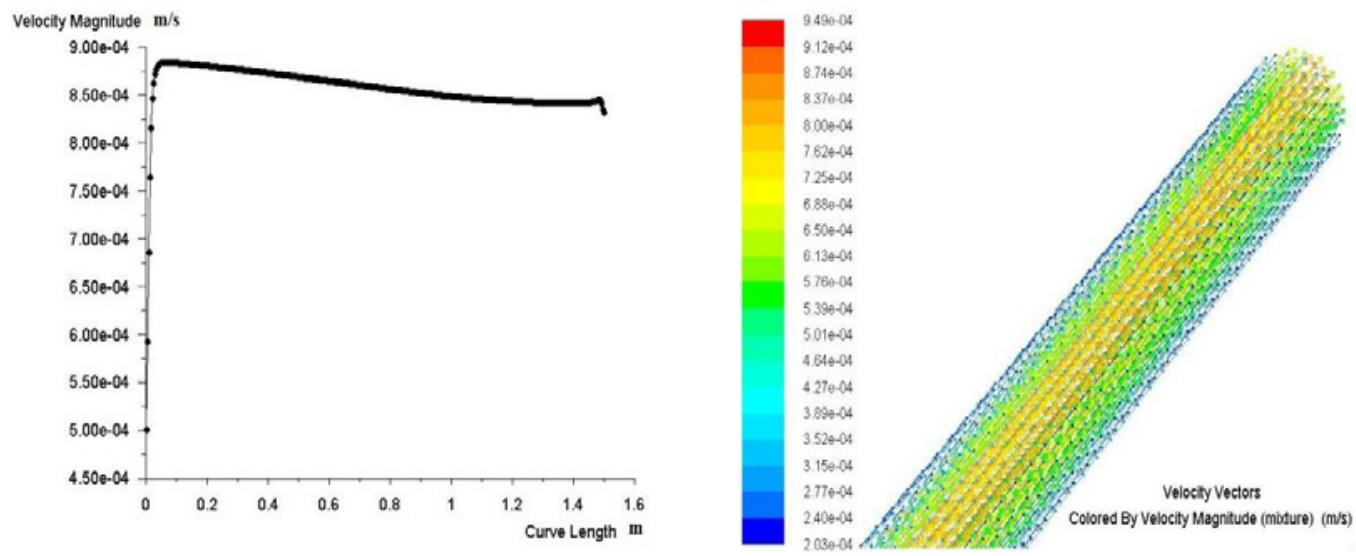

Figure 3. Left results for the velocity magnitude, right velocity vector distribution. 
From the solar calculator the value of direct solar irradiation received to the collector surfaces is $821 \mathrm{~W} / \mathrm{m}^{2}$, and the maximum amount received to the working fluid from is $528 \mathrm{w} / \mathrm{m}^{2}$ indicate that the approximated heat losses is around $293 \mathrm{~W} / \mathrm{m}^{2}$, this amount refer to heat absorption in the copper tube and glass, and the heat loss by convection in the out surface. The rise in the flow water temperature for the selected condition is approximated by $35 \mathrm{~K}$. And the rise in the velocity refers to the increase of the total energy, in the center of the flow.

The simulation results are well agreed with the results published in 2008, by Hyunjoo Han in the study of, a three-dimensional performance analysis of all-glass vacuum tubes with coaxial fluid conduit[7]. Another experimental analysis of thermal performance of flat plate and evacuated tube solar collectors in stationary standard and daily conditions, study done by E. Zambolin 2010 [8], the result obtained in this study is also well validated with Zambolin results.

\section{Conclusions}

The study conclude that the CFD fluent software is a power full tool, that can be used to provide the researchers and scientist in the field of solar collector with accurate results, as a preliminary results that help in taken the right decision in the element design. The results of the evacuated tube collector temperature, and heat losses, are valid with the result published at this field, and the developed evacuated tube collector provide a good solution for solar collector problems.

\section{Acknowledgment}

The authors acknowledge the support from university Teknologi PETRONAS for the research activities, work, and the supply of research facilities.

\section{References}

[1] A. Rabl, Active solar collectors and their applications, New York: Oxford University Press, (1985).

[2] K. V. Karanth, M. Manjunath, and N. Y. Sharma, in Proceedings of the World Congress on Engineering, pp. 6-8, Vol III, (2011).

[3] A. Khalid and H. Junaidi, 45th IEP Convention12, (2012).

[4] K. S. Shashishekar, S. Basavanna, International journal of mechanical engineering and robotic Research Vol. 2, (2013).

[5] A. Venu and P. Arun, International journal of renewable energy research Vol. 3, (2013).

[6] A. F. Ansys, 14.0 User's Guide, ANSYS Inc, (2011).

[7] H. Han, J. T. Kim, H. T. Ahn, and S. J. Lee, International communications in heat and mass Transfer, Vol. 35, pp. 589-596, (2008).

[8] E. Zambolin and D. Del Col, Solar Energy, Vol. 84, pp. 1382-1396, (2010). 